\title{
Characterization by Symmetry of Solutions of a Nonlinear Subelliptic Equation on the Heisenberg Group
}

\author{
Yazhou Han ${ }^{1}$, Xiaodong Wang ${ }^{2}$ and Meijun $\mathrm{Zhu}^{3, *}$ \\ ${ }^{1}$ Department of Mathematics, College of Science, China Jiliang University, Hangzhou, \\ 310018, P.R. China. \\ 2 Department of Mathematics, Michigan State University, East Lansing, MI 48824, \\ USA. \\ ${ }^{3}$ Department of Mathematics, The University of Oklahoma, Norman, OK 73019, \\ USA.
}

Received March 25, 2016; Accepted February 20, 2017

\begin{abstract}
In this paper we prove that two calculus lemmas, which are used in the method of moving sphere for classifying certain constant curvature equation, also hold on Heisenberg group $\mathbb{H}^{n}$.
\end{abstract}

AMS subject classifications: $35 \mathrm{H} 20,22 \mathrm{E} 30$

Key words: Classification, moving sphere method, Heisenberg group.

\section{Introduction}

The Heisenberg group $\mathbb{H}^{n}$ consists of the set

$$
\mathbb{C}^{n} \times \mathbb{R}=\left\{(z, t): z=\left(z_{1}, \cdots, z_{n}\right) \in \mathbb{C}^{n}, t \in \mathbb{R}\right\}
$$

with the multiplication law

$$
(z, t) \circ\left(z^{\prime}, t^{\prime}\right)=\left(z+z^{\prime}, t+t^{\prime}+2 \operatorname{Im}\left(z \cdot \overline{z^{\prime}}\right)\right),
$$

where $z \cdot \overline{z^{\prime}}=\sum_{j=1}^{n} z_{j} \bar{z}_{j}^{\prime}$. As usual, we write $z_{j}=x_{j}+\sqrt{-1} y_{j}$. The Lie algebra is spanned by the left invariant vector fields

$$
T=\frac{\partial}{\partial t}, X_{j}=\frac{\partial}{\partial x_{j}}+2 y_{j} \frac{\partial}{\partial t}, Y_{j}=\frac{\partial}{\partial y_{j}}-2 x_{j} \frac{\partial}{\partial t}, j=1, \cdots, n
$$

${ }^{*}$ Corresponding author. Email addresses: yazhou.han@gmail.com (Y. Han), xwang@math.msu.edu (X. Wang), mzhu@ou.edu (M. Zhu) 
The sub-Laplacian is defined by

$$
\triangle_{H}=\sum_{j=1}^{n}\left(X_{j}^{2}+Y_{j}^{2}\right)
$$

In this paper, we shall study the following semilinear subelliptic equation

$$
-\Delta_{H} u=u^{p}, \quad u \geq 0, \quad \text { in } \mathbb{H}^{n},
$$

where and throughout the paper we always assume that $p>1$.

This equation is closely related to the study of the $C R$ Yamabe problem. The $C R$ Yamabe problem on a strictly pseudonconvex $C R$ manifold is analogous to the original Yamabe problem on compact manifolds. As the first step, Jerison and Lee [8] proved a sharp Sobolev inequality on the CR sphere $\mathbb{S}^{2 n+1}$. Moreover they classified all extremal functions of the sharp Sobolev inequality. Since $S^{2 n+1}$ is CR equivalent to the Heisenberg group via the Cayley transform, their classification can be stated as following.

Theorem 1.1. Let $u \in C^{2}\left(\mathbb{H}^{n}\right)$ be a solution to equation (1.1) with $p=\frac{Q+2}{Q-2}$ (where $Q=2 n+2$ is the homogeneous dimension), and suppose $u \in L^{1+p}$. Then

$$
u(z, t)=\left.|t+\sqrt{-1}| z\right|^{2}+\mu \cdot z+\left.\lambda\right|^{-n},
$$

where $\mu \in \mathbb{C}^{n}, \lambda \in \mathbb{C}$, and $\operatorname{Im} \lambda>|\mu|^{2} / 4$.

The proof hinges on a complicated and remarkable identity they discovered with the help of a computer program. It is motivated by Obata's classic work [16] in the Riemannian case. When restricted on $\mathbb{R}^{n}$, Obata's theomem has the following version.

Theorem 1.2. Let $u \in C^{2}\left(\mathbb{R}^{n}\right)$ be a positive solution for the following equation

$$
-\Delta u=u^{\frac{n+2}{n-2}}
$$

and suppose $u(x)=O\left(|x|^{2-n}\right)$ for large $|x|$. Then

$$
u(x)=\left(\frac{\lambda}{\left|x-x_{0}\right|^{2}+\lambda^{2}}\right)^{(n-2) / 2}
$$

for some $\lambda>0$ and $x_{0} \in \mathbb{R}^{n}$.

We recall that there is another completely different approach to such classification problems. This is the method of moving plane initiated by Gidas, Ni and Nirenberg [6]. In particular they reproved the above theorem using this method. Later, Caffarelli, Gidas and Spruck [4] classified all positive solutions to equation (1.3) by sharpening the method of moving plane. Their result plays a crucial role in establishing the compactness results for the solution set to Yamabe problem, see, e.g., Schoen [17], Li and Zhu [14], 
Li and Zhang [11-13], Kuri, et al. [9], Brendle [3]. More recently Li and Zhu developed moving sphere method and gave a simpler proof of this classification result. Moving sphere method is essentially equivalent to moving plane method, but is more convenient. In particular, a simpler alternative proof for the result of Caffarelli, Gidas and Spruck via the method of moving sphere was given in Li's lecture note [10], and first appeared in Zhu's thesis [19]. In this proof via the method of moving sphere, one fully explores the conformal invariance of the equation and obtains certain conformally invariant identity with respect to any point. Solutions to equation (1.3) can then be derived directly from the identity via two key calculus lemmas (refered as Li-Zhu lemmas [15]).

It is natural question if one can adapt moving plane method or moving sphere method on the Heisenberg group. Such a method might enable us to classify all positive solutions of (1.1) without any integrability condition or growth condition at infinity. More specifically, we have the following conjecture.

Conjecture 1.1. Let $u \in C^{2}\left(\mathbb{H}^{n}\right)$ be a solution to Eq. (1.1). If $p=\frac{Q+2}{Q-2}$ (where $Q=2 n+2$ is the homogeneous dimension), then

$$
u(z, t)=\left.|t+\sqrt{-1}| z\right|^{2}+\mu \cdot z+\left.\lambda\right|^{-n},
$$

where $\mu \in \mathbb{C}^{n}, \lambda \in \mathbb{C}$, and $\operatorname{Im} \lambda>|\mu|^{2} / 4$; If $p \in\left(1, \frac{Q+2}{Q-2}\right)$, then $u(z, t)=0$.

There have been attepmpts by several mathematicians in the direction. The method of moving plane was adapted by Birindelli and Prajapat [2] to study equation (1.1). Under the assumption of cylindrical symmetry (i.e. $u$ only depends on $|z|$ and $t$ ), they proved that the only nonnegative solution to equation (1.1) for subcritical power $1<p<\frac{\mathrm{Q}+2}{\mathrm{Q}-2}$ is the trivial one. The method of moving plane does not work for the general case since it only yields partial invariant properties of solutions. Other partial results for subcritical case were obtained by Birindelli et al. [1], Garofalo and Lanconelli [5] and Xu [18].

Here we shall use the approach based on the method of moving sphere for the conjecture.

For any $\xi=(z, t) \in \mathbb{H}^{n}$, the norm function $\rho(\xi)$ is defined as

$$
\rho(\xi)=\left(|z|^{4}+t^{2}\right)^{\frac{1}{4}} .
$$

It is well known that $d(\eta, \xi):=\rho\left(\eta^{-1} \circ \xi\right)$ defines a distance on $\mathbb{H}^{n}$. On $\mathbb{H}^{n}$ we have the contact 1 -form

$$
\theta=d t+\sqrt{-1} \sum_{j=1}^{n}\left(z_{j} d \bar{z}_{j}-\bar{z}_{j} d z_{j}\right)
$$

The study of the equation (1.1) when $p=(Q+2) /(Q-2)$ must use its invariance under contact transformations on $\mathbb{H}^{n}$ : if $\Phi$ is a diffeomorphism of $\mathbb{H}^{n} \cup \infty$ s.t. $\Phi^{*} \theta=J_{\Phi}^{1 /(n+1)} \theta$, where $J_{\Phi}>0$ is the Jacobian determinant of $\Phi$, and $u$ is a solution of (1.1), then so is $u \circ \Phi J_{\Phi}^{\frac{n}{2(n+1)}}$. Modulo $U(n)$, all the contact transformations of $\mathbb{H}^{n}$ are generated by 
- left translations $L_{\eta}(\xi)=\eta \circ \xi$, with $L_{\eta}^{*} \theta=\theta$,

- dialations $\delta_{\lambda}(z, t)=\left(\lambda z, \lambda^{2} t\right), \lambda>0$, with $\delta_{\lambda}^{*} \theta=\lambda^{2} \theta$,

- the inversion $T(z, t)=\left(\frac{z}{|z|^{2}-\sqrt{-1} t},-\frac{t}{|z|^{4}+t^{2}}\right)$, with $T^{*} \theta=\frac{1}{|z|^{4}+t^{2}} \theta$.

We first exam the solution to (1.1) for $p=(Q+2) /(Q-2)$ with center at the origin:

$$
u(\xi)=\left.|1+| z\right|^{2}+\left.\sqrt{-1} t\right|^{-n} .
$$

Using the left translation operator $L_{\xi_{0}}$, we can obtain the following more general solution

$$
v(\xi):=u \circ L_{\xi_{0}}(\xi)=\left.|| z\right|^{2}+\sqrt{-1} t+2 z_{0} \bar{z}+1+\left|z_{0}\right|^{2}+\left.\sqrt{-1} t_{0}\right|^{-n} .
$$

Using the inversion $T$ and dialation, we know that

$$
v_{\lambda}(\xi):=\left(\frac{\lambda^{4}}{|z|^{4}+t^{2}}\right)^{n / 2} v\left(\delta_{\lambda^{2}} \circ T(\xi)\right)
$$

is also a solution. We compute

$$
\begin{aligned}
v_{\lambda}(\xi)= & v\left(\frac{\lambda^{2} z}{|z|^{2}-\sqrt{-1} t},-\frac{\lambda^{4} t}{|z|^{4}+t^{2}}\right)\left[\frac{\lambda^{2}}{\left.|| z\right|^{2}+\sqrt{-1} t \mid}\right]^{n} \\
= & \left.\left|\frac{\lambda^{4}\left(|z|^{2}-\sqrt{-1} t\right)}{\left.|| z\right|^{2}-\left.\sqrt{-1} t\right|^{2}}+\frac{2 \lambda^{2} z_{0} \bar{z}}{|z|^{2}+\sqrt{-1} t}+1+\right| z_{0}\right|^{2}+\left.\sqrt{-1} t_{0}\right|^{-n}\left[\frac{\lambda^{2}}{\left.|| z\right|^{2}+\sqrt{-1} t \mid}\right]^{n} \\
= & \left.\left|\lambda^{2}+2 z_{0} \bar{z}+\left(1+\left|z_{0}\right|^{2}+\sqrt{-1} t_{0}\right) \frac{|z|^{2}+\sqrt{-1} t}{\lambda^{2}}\right|^{-n}\right|^{-n} \\
= & \left.\left.|| z\right|^{2}+\sqrt{-1} t+\frac{2 \lambda^{2} z_{0} \bar{z}}{\left(1+\left|z_{0}\right|^{2}+\sqrt{-1} t_{0}\right)}+\left.\frac{\lambda^{4}}{\left(1+\left|z_{0}\right|^{2}+\sqrt{-1} t_{0}\right)}\right|^{n}\right]^{n} . \\
& \quad\left[\frac{\lambda^{2}}{\left|\left(1+\left|z_{0}\right|^{2}+\sqrt{-1} t_{0}\right)\right|}\right]^{2}
\end{aligned}
$$

We choose $\lambda=\lambda\left(\xi_{0}\right)=\left.|1+| z_{0}\right|^{2}+\left.\sqrt{-1} t_{0}\right|^{1 / 2}$. Then

$$
\begin{aligned}
v_{\lambda}(\xi) & =\left.|| z\right|^{2}+\sqrt{-1} t+\frac{2 \lambda^{2} z_{0} \bar{z}}{\left(1+\left|z_{0}\right|^{2}+\sqrt{-1} t_{0}\right)}+1+\left|z_{0}\right|^{2}-\left.\sqrt{-1} t_{0}\right|^{-n} \\
& =u \circ L_{\xi_{0}^{\prime}}(\xi),
\end{aligned}
$$


where

$$
\xi_{0}^{\prime}=\left(e^{i \theta_{0}} z_{0},-t_{0}\right), \quad e^{i \theta_{0}}=\frac{1+\left|z_{0}\right|^{2}-\sqrt{-1} t_{0}}{\left.|1+| z_{0}\right|^{2}-\sqrt{-1} t_{0} \mid} .
$$

In summary, we have proved

$$
\left(\frac{\lambda\left(\xi_{0}\right)}{\rho(\xi)}\right)^{2 n} u \circ L_{\xi_{0}} \circ \delta_{\lambda\left(\xi_{0}\right)^{2}} \circ T(\xi)=u \circ L_{\xi_{0}^{\prime}}(\xi),
$$

or, equivalently

$$
\left(\frac{\lambda\left(\xi_{0}\right)}{d\left(\xi_{0}^{\prime}, \xi\right)}\right)^{2 n} u \circ L_{\xi_{0}} \circ \delta_{\lambda\left(\xi_{0}\right)^{2}} \circ T \circ L_{\xi_{0}^{\prime}}^{-1}(\xi)=u(\xi) .
$$

For a general solution $v=u \circ L_{\eta}^{-1}$, this implies that

$$
\left(\frac{\lambda\left(\xi_{0}\right)}{d\left(\eta \xi_{0}^{\prime}, \xi\right)}\right)^{2 n} v \circ L_{\eta \xi_{0}} \circ \delta_{\lambda\left(\xi_{0}\right)^{2}} \circ T \circ L_{\eta \xi_{0}^{\prime}}^{-1}(\xi)=v(\xi) .
$$

Set $\eta_{0}=\eta \xi_{0}, \eta_{0}^{\prime}=\eta \xi_{0}^{\prime}$. We obtain for $\lambda=\lambda\left(\xi_{0}\right)$

$$
\left(\frac{\lambda}{d\left(\eta_{0}^{\prime}, \xi\right)}\right)^{2 n} v \circ L_{\eta_{0}} \circ \delta_{\lambda^{2}} \circ T \circ L_{\eta_{0}^{\prime}}^{-1}(\xi)=v(\xi) .
$$

In this paper, we will show the converse.

Theorem 1.3. For any $\xi_{0}=\left(z_{0}, t_{0}\right) \in \mathbb{H}^{n}$, let $\xi_{0}^{\prime}$ be given by (1.5). If $u \in C^{1}\left(\mathbb{H}^{n}\right)$ satisfies: for all $\lambda>0$,

$$
u(\xi) \geq\left(\frac{\lambda}{d\left(\xi_{0}^{\prime}, \xi\right)}\right)^{v} u \circ L_{\xi_{0}} \circ \delta_{\lambda^{2}} \circ T \circ L_{\xi_{0}^{\prime}}^{-1}(\xi), \quad \forall d\left(\xi_{0}^{\prime}, \xi\right) \geq \lambda>0
$$

holds for a fixed parameter $v$, then $u \equiv$ const.

Theorem 1.4. Assume that $u \in C^{1}\left(\mathbb{H}^{n}\right)$ attaines its maximal value at the origin, and satisfies: for any $\xi_{0}=\left(z_{0}, t_{0}\right) \in \mathbb{H}^{n}$ and $\xi_{0}^{\prime}$ be given by (1.5), there exist a positive constant $\lambda=\lambda\left(\xi_{0}\right)$ such that

$$
u(\xi)=\left(\frac{\lambda}{d\left(\xi_{0}^{\prime}, \xi\right)}\right)^{v} u \circ L_{\tilde{\zeta}_{0}} \circ \delta_{\lambda\left(\xi_{0}\right)^{2}} \circ T \circ L_{\xi_{0}^{\prime}}^{-1}(\xi), \quad \forall \xi \in \mathbb{H}^{n},
$$

holds for a fixed parameter $v$, then $u(\xi)$ is given by the following form

$$
u(\xi)=C\left(\left(|z|^{2}+\lambda(0)^{2}\right)^{2}+t^{2}\right)^{-\frac{v}{4}}=\left.C|| z\right|^{2}+\lambda(0)^{2}+\left.\sqrt{-1} t\right|^{-\frac{v}{2}}
$$

where $C$ is a constant.

Remark 1.5. It is clear that the assumption is invariant under left translation: for any $\eta \in \mathbb{H}^{n}$ the function $v=u \circ L_{\eta}^{-1}$ satisfies the same assumption, though with a different correspondence $\xi_{0} \rightarrow \xi_{0}^{\prime}$. 


\section{Proof}

We first note, by the triangle inequality, that

$$
\frac{d\left(\xi_{0}^{\prime}, \xi\right)^{4}}{\rho(\xi)^{4}}=1+O\left(\rho(\xi)^{-1}\right)
$$

as $\rho(\xi) \rightarrow \infty$. We also have

$$
L_{\tilde{\zeta}_{0}} \circ \delta_{\lambda\left(\tilde{\xi}_{0}\right)^{2}} \circ T \circ L_{\tilde{\xi}_{0}^{\prime}}^{-1}(\xi) \rightarrow \xi_{0}
$$

as $\rho(\xi) \rightarrow \infty$.

Proof of Theorem 1.4. Using (1.7), we define

$$
D:=\lim _{\rho(\xi) \rightarrow+\infty} \rho(\xi)^{v} u(\xi)=\lambda\left(\xi_{0}\right)^{v} u\left(\xi_{0}\right) .
$$

If $D=0$, then $u \equiv 0$, and we are done. Without loss of generality, we assume $D=1$. Further, if $v=0$, then $u \equiv D$, and we are done. On the other hand, the case $v<0$ can easily be reduced to the case of $v>0$ by using a reversed CR inversion. We thus assume that $v>0$ in the following proof.

Taking $\xi_{0}=0$ yields

$$
u(\xi)=\left(\frac{\lambda(0)}{\rho(\xi)}\right)^{v} u \circ \delta_{\lambda(0)^{2}} \circ T(\xi) .
$$

As $u$ attains its maximum at the origin, we have for $\xi$ large

$$
\begin{aligned}
u(\xi) & =\left(\frac{\lambda(0)}{\rho(\xi)}\right)^{v}[u(0)+O(f(\xi))] \\
& =\rho(\xi)^{-v}+\rho(\xi)^{-v} O(f(\xi)),
\end{aligned}
$$

where

$$
f(\xi)=\left|\delta_{\lambda(0)^{2}} T(\xi)\right|^{2}=\frac{\lambda(0)^{4}|z|^{2}}{|z|^{4}+t^{2}}+\frac{\lambda(0)^{8} t^{2}}{\left(|z|^{4}+t^{2}\right)^{2}}
$$

Moreover,

$$
\begin{aligned}
& u(\xi)=\left(\frac{\lambda\left(\xi_{0}\right)}{d\left(\xi_{0}^{\prime}, \xi\right)}\right)^{v} u\left(\xi_{0} \circ \delta_{\lambda\left(\xi_{0}\right)^{2}} \circ T \circ L_{\xi_{0}^{\prime}}^{-1}(\xi)\right) \\
& =\left(\frac{\lambda\left(\xi_{0}\right)}{d\left(\xi_{0}^{\prime}, \xi\right)}\right)^{v}\left[u\left(\xi_{0}\right)+\frac{\partial u}{\partial z}\left(\xi_{0}\right) \lambda\left(\xi_{0}\right)^{2} L_{\xi_{0}^{\prime}}^{-1} \circ \frac{z}{|z|^{2}-\sqrt{-1} t}\right. \\
& +\frac{\partial u}{\partial \bar{z}}\left(\xi_{0}\right) \lambda\left(\xi_{0}\right)^{2} \overline{L_{\xi_{0}^{\prime}}^{-1} \circ \frac{z}{|z|^{2}-\sqrt{-1} t}}+\frac{\partial u}{\partial t}\left(\xi_{0}\right) \lambda\left(\xi_{0}\right)^{4} L_{\xi_{0}^{\prime}}^{-1} \circ \frac{-t}{|z|^{4}+t^{2}} \\
& \left.+\frac{\partial u}{\partial t}\left(\xi_{0}\right) \cdot 2 \lambda\left(\xi_{0}\right)^{2} \operatorname{Im}\left(z_{0} \cdot \overline{L_{\xi_{0}^{\prime}}^{-1} \circ \frac{z}{|z|^{2}-\sqrt{-1} t}}\right)+O(g(\xi))\right],
\end{aligned}
$$


where

$$
\begin{aligned}
g(\xi)=\mid & \left.\lambda\left(\xi_{0}\right)^{2} L_{\xi_{0}^{\prime}}^{-1} \circ \frac{z}{|z|^{2}-\sqrt{-1} t}\right|^{2} \\
& +\mid \lambda\left(\xi_{0}\right)^{4} L_{\xi_{0}^{\prime}}^{-1} \circ \frac{-t}{|z|^{4}+t^{2}}+2 \lambda\left(\xi_{0}\right)^{2} \operatorname{Im}\left(\left.z_{0} \cdot \frac{\left.L_{\xi_{0}^{\prime}}^{-1} \circ \frac{z}{|z|^{2}-\sqrt{-1} t}\right)}{x^{2}}\right|^{2} .\right.
\end{aligned}
$$

Subtracting (2.4) from (2.3), and using (2.1) and (2.2), we have

$$
\begin{aligned}
& d\left(\xi_{0}^{\prime}, \xi^{-v}\left[\lambda\left(\xi_{0}\right)^{v+2} \frac{\partial u}{\partial z}\left(\xi_{0}\right) L_{\xi_{0}^{\prime}}^{-1} \circ \frac{z}{|z|^{2}-\sqrt{-1} t}\right.\right. \\
& +\lambda\left(\xi_{0}\right)^{v+2} \frac{\partial u}{\partial \bar{z}}\left(\xi_{0}\right) \overline{L_{\xi_{0}^{\prime}}^{-1} \circ \frac{z}{|z|^{2}-\sqrt{-1} t}}+\lambda\left(\xi_{0}\right)^{v+4} \frac{\partial u}{\partial t}\left(\xi_{0}\right) L_{\xi_{0}^{\prime}}^{-1} \circ \frac{-t}{|z|^{4}+t^{2}} \\
& \left.+2 \lambda\left(\xi_{0}\right)^{v+2} \frac{\partial u}{\partial t}\left(\xi_{0}\right) \operatorname{Im}\left(z_{0} \cdot \overline{L_{\xi_{0}^{\prime}}^{-1} \circ \frac{z}{|z|^{2}-\sqrt{-1} t}}\right)\right] \\
& +\left[d\left(\xi_{0}^{\prime}, \xi\right)^{-v} O(g(\xi))-\rho(\xi)^{-v} O(f(\xi))\right] \\
& =-d\left(\xi_{0}^{\prime}, \xi\right)^{-v}+\rho(\xi)^{-v} \\
& =-\rho(\xi)^{-v}\left[v \frac{|z|^{2} \operatorname{Re}\left(z_{0} e^{i \theta_{0}} \bar{z}\right)}{\rho(\xi))^{4}}+v \frac{t \operatorname{Im}\left(z_{0} e^{i \theta_{0}} \bar{z}\right)}{\rho(\xi)^{4}}\right. \\
& \left.-\frac{3 v}{2} \frac{\left|z_{0}\right|^{2}|z|^{2}}{\rho(\xi)^{4}}-\frac{v}{2} \frac{t_{0} t}{\rho(\xi)^{4}}+o\left(\rho(\xi)^{-2}\right)\right] .
\end{aligned}
$$

Taking $\xi=\left(z_{0} e^{i \theta_{0}}, t\right)$ in (2.5), we have

$$
\begin{aligned}
& d\left(\xi_{0}^{\prime}, \xi\right)^{-v} \lambda\left(\xi_{0}\right)^{v+4} \frac{\partial u}{\partial t}\left(\xi_{0}\right)\left(-\frac{t+t_{0}}{\left|t+t_{0}\right|^{2}}\right) \\
& \quad+\left[d\left(\xi_{0}^{\prime}, \xi\right)^{-v} O(g(\xi))-\rho(\xi)^{-v} O(f(\xi))\right] \\
& =-\rho(\xi)^{-v}\left[-\frac{v}{2} \frac{\left|z_{0}\right|^{4}}{\left|z_{0}\right|^{4}+t^{2}}-\frac{v}{2} \frac{t_{0} t}{\left|z_{0}\right|^{4}+t^{2}}+o\left(\rho(\xi)^{-2}\right)\right] .
\end{aligned}
$$

Multiplying both sides by $\rho(\xi)^{v+2}$ and letting $t \rightarrow+\infty$, we obtain

$$
\lambda\left(\xi_{0}\right)^{v+4} \frac{\partial u}{\partial t}\left(\xi_{0}\right)=-\frac{v}{2} t_{0}
$$

which yields, by (2.2),

$$
\frac{\partial}{\partial t}\left(u^{-\frac{4}{v}}\right)\left(\xi_{0}\right)=2 t_{0}
$$

It follows that there exists a function $\phi(z, \bar{z})$ such that

$$
u(\xi)=\left(\phi(z, \bar{z})+t^{2}\right)^{-\frac{v}{4}} .
$$


Consequently, we know that $\frac{\partial u}{\partial t}(z, \bar{z}, 0)=0$.

On the other hand, let $\xi_{0}=\left(z_{0}, \overline{z_{0}}, 0\right)=\left(x_{0}, y_{0}, 0\right)$ and $\xi=(z, \bar{z}, 0)=(x, y, 0)$ in $(2.5)$, we have

$$
\begin{aligned}
d\left(\xi_{0}^{\prime}, \xi\right)^{-v} \lambda\left(\xi_{0}\right)^{v+2}\left[\frac{\partial u}{\partial z}\left(\xi_{0}\right) \frac{z-z_{0}}{\left|z-z_{0}\right|^{2}-2 \sqrt{-1}\left(x y_{0}-x_{0} y\right)}\right. \\
\left.+\frac{\partial u}{\partial \bar{z}}\left(\xi_{0}\right) \frac{\bar{z}-\overline{z_{0}}}{\left|z-z_{0}\right|^{2}+2 \sqrt{-1}\left(x y_{0}-x_{0} y\right)}\right] \\
+\left[d\left(\xi_{0}^{\prime}, \xi\right)^{-v} O(g(\xi))-\rho(\xi)^{-v} O(f(\xi))\right] \\
=-\rho(\xi)^{-v}\left[v \frac{|z|^{2} \operatorname{Re}\left(z_{0} \bar{z}\right)}{\rho(\xi)^{4}}-\frac{3 v}{2} \frac{\left|z_{0}\right|^{2}|z|^{2}}{\rho(\xi)^{4}}+o\left(\rho(\xi)^{-2}\right)\right] .
\end{aligned}
$$

Choose $y=0$ in (2.7). Multiplying both sides by $\rho(\xi)^{v+1}$, and sending $x \rightarrow+\infty$, we get

$$
\lambda\left(\xi_{0}\right)^{v+2}\left[\frac{\partial u}{\partial z}\left(\xi_{0}\right)+\frac{\partial u}{\partial \bar{z}}\left(\xi_{0}\right)\right]=-v x_{0}
$$

Similarly, choosing $x=0$ in (2.7), we obtain

$$
-\sqrt{-1} \lambda\left(\xi_{0}\right)^{v+2}\left[\frac{\partial u}{\partial z}\left(\xi_{0}\right)-\frac{\partial u}{\partial \bar{z}}\left(\xi_{0}\right)\right]=v y_{0}
$$

Combining (2.8) with (2.9), we have

$$
\lambda\left(\xi_{0}\right)^{v+2} \frac{\partial u}{\partial z}\left(\xi_{0}\right)=-\frac{v}{2} \overline{z_{0}}, \quad \lambda\left(\xi_{0}\right)^{v+2} \frac{\partial u}{\partial \bar{z}}\left(\xi_{0}\right)=-\frac{v}{2} z_{0}
$$

This yields, that $\phi(z, \bar{z})$ in (2.6) satisfies

$$
\frac{\partial \sqrt{\phi(z, \bar{z})}}{\partial z}=\bar{z}, \quad \frac{\partial \sqrt{\phi(z, \bar{z})}}{\partial \bar{z}}=z .
$$

Therefore, we conclude that there exists a constant $C_{*}$ such that

$$
u(\xi)=\left(\left(|z|^{2}+C_{*}\right)^{2}+t^{2}\right)^{-\frac{v}{4}} .
$$

In fact, from (2.2), we know $C_{*}=\lambda(0)^{2}$.

Proof of Theorem 1.3. Step I: We first show that $u(\xi)=u(z, \bar{z})$, that is, function $u(\xi)$ is independent of variable $t$.

For any given $t, \tau \in \mathbb{R}(t \neq \tau)$ and any $b>1$, let $\xi=\left(z_{0}, \overline{z_{0}}, t\right), \zeta=\left(z_{0}, \overline{z_{0}}, \tau\right) \in \mathbb{H}^{n}$,

$$
t_{0}=t_{0}(b)=-\tau+b(t+\tau) \quad \text { and } \quad \lambda=\lambda(b)=\sqrt{d\left(\xi_{0}^{\prime}, \zeta\right) d\left(\xi_{0}, \zeta\right)}
$$


where $\xi_{0}=\left(0,0, t_{0}\right)$ and $\xi_{0}^{\prime}=\left(0,0,-t_{0}\right)$. A direct computation leads to

$$
\begin{aligned}
& d\left(\xi_{0}, \xi\right)=\left(\left|z_{0}\right|^{4}+(b-1)^{2} \cdot|t+\tau|^{2}\right)^{\frac{1}{4}}, \\
& d\left(\xi_{0}^{\prime}, \zeta\right)=\left(\left|z_{0}\right|^{4}+b^{2} \cdot|t+\tau|^{2}\right)^{\frac{1}{4}} \\
& \lambda^{2}=\left(\left|z_{0}\right|^{4}+(b-1)^{2} \cdot|t+\tau|^{2}\right)^{\frac{1}{4}}\left(\left|z_{0}\right|^{4}+b^{2} \cdot|t+\tau|^{2}\right)^{\frac{1}{4}}<d\left(\xi_{0}^{\prime}, \zeta\right)^{2} .
\end{aligned}
$$

Thus, we know, via (1.6), that

$$
u(\zeta) \geq\left(\frac{\lambda}{d\left(\xi_{0}^{\prime}, \zeta\right)}\right)^{v} u\left(\xi_{0} \circ \delta_{\lambda^{2}} \circ T \circ L_{\xi_{0}^{\prime}}^{-1}(\zeta)\right)
$$

Noting that

$$
\begin{aligned}
& \xi_{0} \circ \delta_{\lambda^{2}} \circ T \circ L_{\xi_{0}^{\prime}}^{-1}(\zeta)=\left(\frac{\lambda^{2} z_{0}}{\left|z_{0}\right|^{2}-i b(t+\tau)^{\prime}}, \frac{\lambda^{2} \overline{z_{0}}}{\left|z_{0}\right|^{2}+i b(t+\tau)^{\prime}}, t_{0}-\frac{\lambda^{4} b(t+\tau)}{\left|z_{0}\right|^{4}+b^{2}|t+\tau|^{2}}\right), \\
& \lim _{b \rightarrow+\infty} \frac{\lambda}{d\left(\xi_{0}^{\prime}, \zeta\right)}=1, \\
& \lim _{b \rightarrow+\infty} \frac{\lambda^{2} z_{0}}{\left|z_{0}\right|^{2}-i b(t+\tau)}= \begin{cases}\sqrt{-1} z_{0}, & \text { if } t+\tau>0, \\
-\sqrt{-1} z_{0}, & \text { if } t+\tau<0,\end{cases} \\
& t_{0}-\frac{\lambda^{4} b(t+\tau)}{\left|z_{0}\right|^{4}+b^{2}|t+\tau|^{2}}=-\tau+\frac{b(t+\tau)}{\left(\left|z_{0}\right|^{4}+b^{2}(t+\tau)^{2}\right)^{\frac{1}{2}}} \\
& \quad \cdot\left(\left(\left|z_{0}\right|^{4}+b^{2}(t+\tau)^{2}\right)^{\frac{1}{2}}-\left(\left|z_{0}\right|^{4}+(b-1)^{2}(t+\tau)^{2}\right)^{\frac{1}{2}}\right) \\
& =-\tau+\frac{b(t+\tau)}{\left(\left|z_{0}\right|^{4}+b^{2}(t+\tau)^{2}\right)^{\frac{1}{2}}} \cdot \frac{s(t+\tau)^{2}}{\left(\left|z_{0}\right|^{4}+s^{2}(t+\tau)^{2}\right)^{\frac{1}{2}}}(b-1<s<b) \\
& \rightarrow-\tau+(t+\tau)=t \quad(\text { as } b \rightarrow+\infty),
\end{aligned}
$$

we obtain, by letting $b \rightarrow+\infty$ in (2.10), that

$$
\begin{cases}u\left(z_{0}, \overline{z_{0}}, \tau\right) \geq u\left(\sqrt{-1} z_{0},-\sqrt{-1} \overline{z_{0}}, t\right), & \text { if } t+\tau>0, \\ u\left(z_{0}, \overline{z_{0}}, \tau\right) \geq u\left(-\sqrt{-1} z_{0}, \sqrt{-1} \overline{z_{0}}, t\right) & \text { if } t+\tau<0 .\end{cases}
$$

For any $t_{1}, t_{2} \in \mathbb{R}$, we choose $t_{3} \in \mathbb{R}$ such that $t_{1}+t_{3}>0$ and $t_{2}+t_{3}>0$. Repeating the above process, we have

$$
\begin{aligned}
u\left(z_{0}, \overline{z_{0}}, t_{1}\right) & \geq u\left(\sqrt{-1} z_{0},-\sqrt{-1} \overline{z_{0}}, t_{3}\right) \geq u\left(-z_{0},-\overline{z_{0}}, t_{2}\right) \\
& \geq u\left(-\sqrt{-1} z_{0}, \sqrt{-1} \overline{z_{0}}, t_{3}\right) \geq u\left(z_{0}, \overline{z_{0}}, t_{2}\right) .
\end{aligned}
$$

Thus, $u$ is independent on the variable $t$ because of the arbitrariness of $t_{1}$ and $t_{2}$.

Step II: We show $u(\xi)=u\left(|z| e^{i \theta},|z| e^{-i \theta}, t\right)=u\left(e^{i \theta}, e^{-i \theta}\right):=\varphi(\theta)$. 
For given two different positive numbers $b, c$ (with $b \neq c$ ) and any $s>1$, choose $a=$ $a(s)=c+s(b-c)$. Let $\xi_{0}=\left(a z_{0}, a \overline{z_{0}}, 0\right), \xi=\left(b z_{0}, b \overline{z_{0}}, 0\right)$ and $\zeta=\left(c z_{0}, c \overline{z_{0}}, 0\right)$. Then $\xi_{0}^{\prime}=$ $\left(a z_{0}, a \overline{z_{0}}, 0\right)$. let

$$
\lambda=\lambda(s)=\sqrt{d\left(\xi_{0}, \xi\right) d\left(\xi_{0}^{\prime}, \zeta\right)}
$$

A direct computation yields

$$
\begin{aligned}
& d\left(\xi_{0}, \xi\right)=\left|(1-s)(b-c) z_{0}\right|, \quad d\left(\xi_{0}^{\prime}, \zeta\right)=\left|s(b-c) z_{0}\right|, \\
& \lambda=\sqrt{|s(1-s)| \mid}(b-c) z_{0} \mid .
\end{aligned}
$$

Thus (using the fact $s>1$ )

$$
\begin{aligned}
& \xi_{0} \circ \delta_{\lambda^{2}} \circ T \circ L_{\xi_{0}^{\prime}}^{-1}(\zeta)=\xi, \quad d\left(\xi_{0}, \xi\right)<\lambda<d\left(\xi_{0}^{\prime}, \zeta\right), \\
& \lim _{s \rightarrow+\infty} \frac{\lambda}{d\left(\xi_{0}^{\prime}, \zeta\right)}=1 .
\end{aligned}
$$

So, by (1.6), we have

$$
u\left(c z_{0}, c \overline{z_{0}}, 0\right) \geq\left(\frac{\lambda}{d\left(\tilde{\zeta}_{0}^{\prime}, \zeta\right)}\right)^{v} u\left(b z_{0}, b \overline{z_{0}}, 0\right)
$$

Sending $s \rightarrow+\infty$, we have $u\left(c z_{0}, c \overline{z_{0}}, 0\right) \geq u\left(b z_{0}, b \overline{z_{0}}, 0\right)$, that is, $u(z, \bar{z}, 0)$ is independent of $|z|$.

Step III: Finally, we show $\varphi(\theta) \equiv$ const.

By (1.6), we know that, for any two positive constants $r, t$ satisfying $r^{4}+t^{2} \geq \lambda^{4}>0$,

$$
\begin{aligned}
\varphi(\theta) & =u\left(r e^{i \theta}, r e^{-i \theta}, t\right) \\
& \geq\left(\frac{\lambda^{4}}{r^{4}+t^{2}}\right)^{\frac{v}{4}} u\left(\delta_{\lambda^{2}} \circ T\left(r e^{i \theta}, r e^{-i \theta}, t\right)\right) \\
& =\left(\frac{\lambda^{4}}{r^{4}+t^{2}}\right)^{\frac{v}{4}} u\left(\frac{\lambda^{2} r e^{i \theta}}{r^{2}-i t}, \frac{\lambda^{2} r e^{-i \theta}}{r^{2}+i t}, \frac{-\lambda^{4} t}{r^{4}+t^{2}}\right) \\
& =\left(\frac{\lambda^{4}}{r^{4}+t^{2}}\right)^{\frac{v}{4}} \varphi\left(\theta+\theta^{\prime}\right),
\end{aligned}
$$

where $\theta^{\prime}=\arctan \frac{t}{r^{2}}$. In particular, choosing $\lambda^{4}=r^{4}+t^{2}$, we have

$$
\varphi(\theta) \geq \varphi\left(\theta+\theta^{\prime}\right)
$$

Since $r, t$ are arbitrary, we conclude that $\varphi(\theta) \equiv$ const. 


\section{Acknowledgments}

The work of Y. Han is partially supported by the National Natural Science Foundation of China (11201443) and Natural Science Foundation of Zhejiang Province (Y6110118).

\section{References}

[1] I. Birindelli, I.C. Dolcetta, A. Cutri. Liouville theorems for semilinear equations on the Heisenberg groups. Ann. I.H.P., 1997, 14: 295-308.

[2] I. Birindelli, J. Prajapat. Nonlinear Liouville theorems in the Heisenberg group via the moving plane method. Comm. P.D.E., 1999, 24: 1875-1890.

[3] S. Brendle. Blow up phenomena for the Yamabe equation. J. Amer. Math. Soc., 2008, 21(4): 951-979.

[4] L. Caffarelli, B. Gidas, J. Spruck. Asymptotic symmetry and local behavior of semilinear elliptic equations with critical Sobolev growth. Comm. Pure Appl. Math., 1989, 42(3): 271297.

[5] N. Garofalo, E. Lanconelli. Existence and nonexistence results for semilinear equations on the Heisenberg group. Indiana Univ. Math. J., 1992, 41(1): 71-98.

[6] B. Gidas, W. Ni, L. Nirenberg. Symmetry and Related Properties via the Maximum Principle. Communications in Mathematical Physics, 1979, 68: 209-243.

[7] B. Gidas, J. Spruck. Global and local behavior of positive solutions of nonlinear elliptic equations. Comm. Pure Appl. Math., 1981, 85: 525-598.

[8] D.S. Jerison, J.M. Lee. Extremals for the Sobolev inequality on the Heisenberg group and Yamabe Problem. J. Amer. Math. Soc., 1988, 1: 1-13.

[9] M.A. Khuri, F.C. Marques, R. Schoen. A compactness theorem for the Yamabe problem. J. Differential Geom. 2009, 81(1): 143-196.

[10] Y.Y. Li. Topics in Analysis. Rutgers University, Personal Note, 1994.

[11] Y.Y. Li, L. Zhang. A Harnack type inequality for the Yamabe equation in low dimensions. Calc. Var. Partial Differential Equations, 2004, 20(2): 133-151.

[12] Y.Y. Li, L. Zhang. Compactness of solutions to the Yamabe problem II. Calc. Var. PDEs,., 2005, 25: 185-237.

[13] Y.Y. Li, L. Zhang. Compactness of solutions to the Yamabe problem III. J. Funct. Anal., 2006, 245: 438-474.

[14] Y.Y. Li, M.J. Zhu. Yamabe type equations on three dimensional Riemannian manifolds. Communications in Contemporary Math., 1999, 1: 1-50.

[15] Y.Y. Li, M.J. Zhu. Uniqueness theorems through the method of moving spheres. Duke Math. J., 1995, 80(2): 383-417.

[16] M. Obata. The conjecture on conformal transformations of Riemannian manifolds. J. Differential Geom., 1971, 6: 247-258.

[17] R. Schoen. Variational theory for the total scalar curvature functional for Riemannian metrics and related topics. Topics in calculus of variations. Springer Berlin Heidelberg, 1989: 120154.

[18] L. Xu. Semi-linear Liouville theorems in the Heisenberg group via vector field methods. J.Differential Equations., 2009, 247: 2799-2820.

[19] M. Zhu. Moving sphere method and sharp Sobolev inequality. Ph.D Dissertation, Rutgers University, 1996. 